\title{
Preoperative cardiopulmonary exercise testing for risk assessment before elective coronary artery bypass grafting surgery
}

\author{
Mariana Carazza, Fernando CC Souza, Andrea De Lorenzo
}

Research and Teaching department, National Institute of Cardiology, Rio de Janeiro, RJ, Brazil

\begin{abstract}
Objective: To evaluate the feasibility and results of performing cardiopulmonary exercise test (CPET) in coronary artery disease (CAD) patients before elective coronary artery bypass grafting surgery (CABG). CPET has been increasingly employed in the evaluation of preoperative risk before noncardiac surgeries, but is still underutilized before cardiac surgery and may be useful, as clinical risk scores for preoperative assessment before cardiac surgeries have several limitations.

Methods: Patients with CAD underwent CPET within 1 week before CABG. Oxygen consumption, oxygen pulse, oxygen consumption at the anaerobic threshold, minute ventilation/carbon dioxide relationship slope $\left(\mathrm{VE} / \mathrm{VCO}_{2}\right.$ slope), oxygen uptake efficiency slope (OUES) and heart rate recovery (HRR) were analyzed. The occurrence of complications during the test was recorded.

Results: Twenty-eight patients (75\% men), aged 61 (8) years, underwent preoperative CPET. There were no complications during CPET, even though $71.4 \%$ were interrupted by signs or symptoms of ischemia, and only $57 \%$ of the patients reached the anaerobic threshold.

Conclusions: Preoperative CPET before elective CABG was feasible and safe. However, a large proportion of patients did not achieve the anaerobic threshold. Therefore, $\mathrm{VE} / \mathrm{VCO}{ }_{2}$ slope, OUES and HRR may be the most useful CPET variables in the preoperative period.
\end{abstract}

Key words: coronary artery disease, coronary artery bypass grafting, cardiopulmonary exercise test, preoperative assessment

(Heart Vessels Transplant 2021; 5: doi: 10.24969/hvt.2021.284)

\section{Introduction}

Coronary artery disease (CAD) is the leading cause of death worldwide (1), and coronary artery bypass grafting surgery (CABG), a high-complexity procedure, is one of the options for its management of CAD. In this context, in addition to the development and improvement of surgical techniques and postoperative care, accurate preoperative evaluation is necessary for better planning by the heart team and optimization of surgical results.

The evaluation of the preoperative risk in cardiac surgery has been based, for over 35 years, on the use of risk scores (2). However, these models have problems related to discrimination and calibration (3). Cardiopulmonary exercise testing (CPET) has been studied as an additional tool in the preoperative assessment for various surgical settings (4). Variables such as oxygen uptake during peak exercise (VO2 peak), slope of the ventilatory equivalent for carbon dioxide (VE/VCO2 slope), oxygen uptake efficiency slope (OUES) and anaerobic threshold (AT) have been widely described as predictors of complications in pulmonary, abdominal, urological and vascular surgeries (5).

In cardiac surgery, some studies have correlated low levels of physical activity, evaluated through questionnaires, with complications in the postoperative period of cardiac surgery (6).

However, the use of indirect measures to assess functional capacity in cardiac patients has numerous limitations, especially in patients with multiple comorbidities, the elderly and patients unable to perform maximum tests. The CPET, in turn, is the method of choice for the functional evaluation of patients with heart disease not only because it directly measures oxygen uptake but also because it provides other prognostic variables obtained in maximal or submaximal tests.

Address for correspondence: Andrea De Lorenzo, Instituto Nacional de Cardiologia, Research and Teaching department, Rua das Laranjeiras 374, Rio de Janeiro, RJ, Brazil, ZIP code 22240-006 Phone: 552130372288 Email: andlorenzo@hotmail.com, andlorenzo@cardiol.br 
Given the growing evidence that low functional capacity is associated with worse outcomes in surgical patients and that the indirect assessment of oxygen uptake may not be adequate in cardiac patients, in addition to the increased use of CPET in the preoperative evaluation for non-cardiac surgery, it is possible that the inclusion of CPET in the preoperative evaluation for CABG can refine risk assessment in this group of patients.

This study aimed to evaluate the feasibility of performing CPET in patients with CAD scheduled for elective $C A B G$ and to describe the complications of this test in this population.

\section{Methods}

\section{Study design and population}

This was a prospective, observational cohort study, which evaluated 28 patients $\geq 18$ years, with stable CAD, who were admitted to a quaternary hospital in Rio de Janeiro, Brazil, for elective, isolated CABG. The study was approved by the Ethics Committee of the National Institute of Cardiology (\# 67629917.1.0000.5272) and registered at ClinicalTrials.org (NCT03376542). All patients signed an informed consent. Exclusion criteria were: 1) Canadian Cardiovascular Society class IV angina; 2) class IV New York Heart Association functional class; 3) mobility restrictions that resulted in an inability to walk on a treadmill; or 4) CABG combined with any other unplanned cardiac intervention, such as valve repairs, thoracic aortic manipulation or left ventricular aneurysmectomy.

\section{Baseline clinical variables}

The following demographic, anthropometric and clinical variables were recorded: age, sex, weight, height, creatinine clearance calculated by the Cockcroft-Gault formula, left ventricular ejection fraction, history of heart failure, history of current or previous smoking, arterial hypertension, or previous myocardial infarction, presence of cerebrovascular disease, peripheral arterial disease. The number of coronary arteries affected by obstructive lesions (luminal obstruction $>70 \%$ ) was defined according to the coronary angiography used by the assistant team for surgical planning.

\section{CPET}

Patients underwent CPET using a treadmill

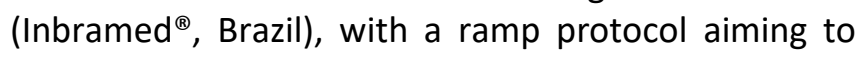
achieve exhaustion within 10 minutes of exercise. The test was limited by symptoms or signs of ischemia or clinical deterioration, such as ST segment depression equal to or greater than $3 \mathrm{~mm}$ relative to the amplitude observed on the resting electrocardiogram, systolic blood pressure drop > $10 \mathrm{mmHg}$ after the third minute of exercise, development of sustained arrhythmias, and signs of imminent risk of fall, among others. During CPET, patients were continuously monitored with a 13-lead electrocardiogram (Ergo PC

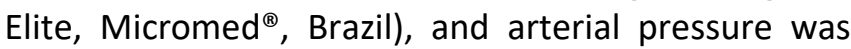
measured every two minutes using a manual sphygmomanometer (Riester ${ }^{\circledR}$, Germany).

Heart rate reduction in the first minute of recovery (HRR) was calculated as the difference between heart rate measured in the first minute of active recovery at $2.0 \mathrm{~km} / \mathrm{h}$ and without incline and heart rate measured at peak exercise. Respiratory gases were reported as mean every 10 seconds using a gas analyser (VO2000, MedGraphics ${ }^{\circledR}$, USA). Before each test, the instrument was calibrated according to the manufacturer's recommendations. The patients used a mouthpiece connected to a pneumotachograph, and expired gas data were collected at rest, during an adaptation phase to the treadmill and warm up at a pace of 2.0 $\mathrm{km} / \mathrm{h}$, during exercise and in the recovery phase. Oxygen uptake during peak exercise (VO2 peak) was considered the highest value obtained between the 30 seconds preceding peak exercise and the first 10 seconds of recovery, and the predicted uptake was calculated taking into account sex, age and weight.

AT was expressed as the oxygen uptake corresponding to the moment at which one of the following occurred: 1) a consistent increase in the ventilatory equivalent for oxygen (VE/VO2) without a corresponding increase in the ventilatory equivalent for carbon dioxide (VE/VCO2); 2) an increase in the fraction of expired oxygen without a simultaneous decrease in the fraction of expired carbon dioxide or 3) a modification in the linear relationship between VO2 and VCO2 (V slope method). The peak VCO2/VO2 (RQ) was defined as the highest value obtained during the last 30 seconds of exercise.

The VE and VO2 values acquired from the beginning to the end of exercise (excluding the values obtained during adaptation and warm up) were entered into calculation software $\left(\right.$ Excel $^{\circledR}, \mathrm{Microsoft}^{\circledR}$, USA) to obtain the OUES. The slope of the ventilatory equivalent for carbon dioxide (VE/VCO2 slope) was also obtained from the beginning to the end of exercise by linear regression between ventilation (VE) and the volume of expired $\mathrm{CO} 2$ (VCO2). Heart rate recovery (HRR) at 1-minute post-exercise was subtracted from maximal heart rate during the exercise test to produce a measure in beats per minute. The tests were performed by a single researcher who was blinded to the clinical characteristics of the patients. 


\section{Statistical analysis}

Continuous variables were expressed as mean and standard deviation and compared by Student's t-test. Categorical variables were compared using the Chisquare test. A value of $5 \%$ was adopted for statistical significance.

\section{Results}

Epidemiological and clinical data are summarized in Table 1. Most patients were male, with mean age of 61 years, and a high prevalence of cardiovascular risk factors was observed. While the majority of patients had left main or 3-vessel CAD, left ventricular ejection fraction was overall normal.

\begin{tabular}{|l|l|}
\hline \multicolumn{2}{|l|}{ Table 1. Demographic and clinical data } \\
\hline & $\mathrm{n}(\%)$ or mean (SD) \\
\hline Male gender & $21(75 \%)$ \\
\hline Age, years & $61(8)$ \\
\hline $\mathrm{BMI}, \mathrm{kg} / \mathrm{m}^{2}$ & $27.1(4.2)$ \\
\hline $\mathrm{CrCl}, \mathrm{ml} / \mathrm{min}$ & $83.2(28.6)$ \\
\hline Ejection fraction, \% & $55.9(13.5)$ \\
\hline Symptoms of heart failure, $\mathrm{n}(\%)$ & $11(39.3)$ \\
\hline Myocardial infarction, $\mathrm{n}(\%)$ & $19(67.9)$ \\
\hline Diabetes, $\mathrm{n}(\%)$ & $13(46.4)$ \\
\hline Hypertension, $\mathrm{n}(\%)$ & $25(89.3)$ \\
\hline Smoking, $\mathrm{n}(\%)$ & $13(48.1)$ \\
\hline Cerebrovascular disease, $\mathrm{n}(\%)$ & $3(10.3)$ \\
\hline Obstructive peripheral arterial disease, $\mathrm{n}(\%)$ & $2(6.9)$ \\
\hline 3-vessel CAD or LMCA, $\mathrm{n}(\%)$ & $23(82.1)$ \\
\hline $\begin{array}{l}\text { BMl - body mass index; CAD -coronary artery disease; } \mathrm{CrCl} \text { - creatinine } \\
\text { clearance; LMCA - left main coronary artery }\end{array}$ \\
\hline
\end{tabular}

\section{Table 2. Cardiopulmonary exercise testing results}

\begin{tabular}{|c|c|}
\hline Variables & Mean (SD) \\
\hline $\mathrm{RQ}$ & $1.03(0.11)$ \\
\hline Peak $\mathrm{VO}_{2}, \mathrm{~mL} \mathrm{~kg}^{-1} \cdot \mathrm{min}^{-1}$ & $13.7(3.1)$ \\
\hline Predicted $\mathrm{VO}_{2}, \%$ & 46.0 ( 12.8) \\
\hline Pulse $\mathrm{O}_{2}, \mathrm{~mL} \mathrm{bpm}^{-1}$ & $10.2(2.8)$ \\
\hline $\mathrm{VO}_{2} \mathrm{AT}, \mathrm{mL} \cdot \mathrm{kg}^{-1} \cdot \mathrm{min}^{-1 *}$ & $11.9(2.6)$ \\
\hline OUES & 1488 (418) \\
\hline $\mathrm{VE} / \mathrm{VCO}_{2}$ slope & $23.5(4.2)$ \\
\hline $\mathrm{HRR}, \mathrm{bpm}$ & $11.5(7.3)$ \\
\hline Peak DP & $15258(3504)$ \\
\hline \multicolumn{2}{|c|}{$\begin{array}{l}\text { bpm - beats per minute, HRR - heart rate reduction in the first minute of recovery, OUES - oxygen uptak } \\
\text { efficient slope, peak VO2 - oxygen uptake at peak exercise, peak DP - double product achieved at peak o } \\
\text { exercise, predicted VO2 - percentage of oxygen uptake predicted, pulse O2 - pulse oxygen; RQ - respirator } \\
\text { exchange ratio, VO2AT - oxygen uptake at the anaerobic threshold, VE/VCO2 slope - slope of the ventilator } \\
\text { equivalent for CO2 } \\
\text { * among } 15 \text { patients }\end{array}$} \\
\hline
\end{tabular}


Complications were not observed in any of the tests. Twenty tests (71.4\%) were interrupted due to signs or symptoms of myocardial ischemia: eleven (39.3\%) due to chest pain and nine $(32.1 \%)$ due to electrocardiographic or hemodynamic criteria. The other eight tests (28.6\%) were interrupted by respiratory fatigue or lower limb fatigue. The AT could only be identified in $57 \%$ of tests and only in nine $(45 \%)$ tests among those interrupted due to ischemia. The mean RQ of the tests was $1.03(0.11)$. Peak $\mathrm{VO}_{2}$ was significantly lower than expected, reaching an average lower than $50 \%$ of predicted, in agreement with the lower $R Q$, possibly due to the large number of submaximal tests. The double product was also reduced, as expected in fully medicated individuals. The $\mathrm{VE} / \mathrm{VCO}_{2}$ slope was within the values considered normal, while OUES and HRR presented values slightly below normal. The main data obtained in the CPET are shown in Table 2.

\section{Discussion}

There is a paucity of data regarding the use of CPET in the preoperative evaluation for CABG. One of the reasons for this may be the fact that patients with major CAD who meet criteria for surgical revascularization (most symptomatic, with large ischemic areas or with left ventricular dysfunction), are considered high risk for exercise tests. In this study, although $71.4 \%$ of the tests were discontinued due to signs or symptoms of myocardial ischemia, no complications were observed during the tests.

The large number of early interruptions of CPET resulted in a mean $R Q$ of $1.03(0.11)$. Due to the large number of submaximal tests, the AT was identified in only $57 \%$ of patients, and the VO2 peak was underestimated. Therefore, these two variables, which are considered important predictors in surgical cohorts (7), may not be useful in the prognostic evaluation of patients in the CABG preoperative period.

On the other hand, ventilatory efficiency, evaluated using VE/VCO2 slope, may be employed even in tests with an $R Q<1.0$ (8) and has been considered a predictor of events in pulmonary surgery (9). Also, the OUES has been considered an easily obtained, reproducible, submaximal variable with a proven prognostic value in the preoperative period of pulmonary surgery $(10,11)$; therefore, OUES may also be used as an accurate measure of cardiopulmonary reserve even in patients who do not reach an $R Q>1.0$ (12).

Finally, HRR after exercise, which prognostic value has been confirmed in submaximal tests (13), has proven prognostic value in patients with lung cancer undergoing lung resection (14).

Thus, we believe that in CAD patients, other CPET variables such as VE/VCO2 slope, OUES and HRR, may be the most useful CPET variables in the preoperative period.

\section{Limitations of the study}

Several limitations of our study should be noted. This is a single-center study with a small number of participants. The sample showed great variation in age and left ventricular systolic function, variables that can significantly influence the results obtained in the CPET.

\section{Conclusions}

This preliminary study shows that CPET before elective CABG is a safe procedure, which may provide interpretable submaximal variables such as OUES, $\mathrm{VE} / \mathrm{VCO}_{2}$ slope and HRR. Larger studies may further define the prognostic value of these variables, validating the use of CPET as a preoperative assessment tool in this population.

Peer-review: External and internal Conflict of interest: None to declare

Authorship: All authors were involved in the conception and design of the study. F.C.C.S. performed the CPET tests. M.C. drafted the manuscript which was revised by F.C.C.S and A.L. The latter was responsible for the final version of the manuscript. All authors approved the final version for submission

Acknowledgement: The authors thank Dr Alexandre Colafranceschi for important discussions regarding the results.

Funding: None to declare

\section{References}

1. Virani SS, Alonso A, Benjamin EJ, Bittencourt MS, Callaway CW, Carson AP, et al. Heart Disease and Stroke Statistics-2020 Update: A Report From the American Heart Association, Circulation 2020; 141: e139-e596.

2. Nashef SAM, Roques F, Michel P, Gauducheau E, Lemeshow S, Salamon R. European system for cardiac operative risk evaluation (Euro SCORE). Eur J Cardiothoracic Surg 1999; 16: 9-13.

3. Garofallo SB, Machado DP, Rodrigues CG, Bordim O, Kalil RAK, Portal VL. Applicability of two international risk scores in cardiac surgery in a reference center in Brazil. Arq Bras Cardiol 2014; 102: 539-48. 
4. Iorio A, Magrì D, Paolillo S, Salvioni E, Di Lenarda A, Sinagra G, et al. Rationale for cardiopulmonary 5. Levett DZH, Grocott MPW. Cardiopulmonary exercise testing for risk prediction in major abdominal surgery. Anesthesiol Clin 2015; 33: 1-16.

6. van Laar C, TImman ST, Noyez L. Decreased physical activity is a predictor for a complicated recovery post cardiac surgery. Health Qual Life Outcomes 2017; 15: 5.

7. Older P. Anaerobic threshold, is it a magic number to determine fitness for surgery? Perioper Med 2013; 2: 2.

8. Chase PJ, Kenjale A, Cahalin LP, Arena R, Davis PG, Myers J, et al. Effects of respiratory exchange ratio on the prognostic value of peak oxygen consumption and ventilatory efficiency in patients with systolic heart failure. JACC Heart Fail 2013; 1: 427-32.

9. Eapen MS, Grover R, Ahuja K, Williams A, Sohal SS. Ventilatory efficiency slope as a predictor of suitability for surgery in chronic obstructive pulmonary disease patients with lung cancer. Ann Transl Med 2016; 4: 296-296.

10. Van Laethem C, De Sutter J, Peersman W, Calders $P$. Intratest reliability and test-retest reproducibility of exercise test in the assessment of surgical risk. J Cardiovasc Med 2013; 14: 254-61.

the oxygen uptake efficiency slope in healthy participants. Eur J Cardiovasc Prev Rehabil 2009; 16: 493-8.

11. Davies LC, Wensel R, Georgiadou P, Cicoira M, Coats AJS, Piepoli MF, et al. Enhanced prognostic value from cardiopulmonary exercise testing in chronic heart failure by non-linear analysis: Oxygen uptake efficiency slope. Eur Heart J 2006; 27: 684-90.

12. Hollenberg M, Tager IB. Oxygen uptake efficiency slope: an index of exercise performance and cardiopulmonary reserve requiring only submaximal exercise. J Am Coll Cardiol 2000; 36: 194-201.

13. Cahalin LP, Forman DE, Chase P, Guazzi M, Myers $J$, Bensimhon $D$, et al. The prognostic significance of heart rate recovery is not dependent upon maximal effort in patients with heart failure. Int J Cardiol 2013; 168: 1496-501.

14. Ha D, Choi H, Zell K, Raymond DP, Stephans K, Wang XF, et al. Association of impaired heart rate recovery with cardiopulmonary complications after lung cancer resection surgery. J Thorac Cardiovasc Surg 2015; 149: 1168-1173.e3.

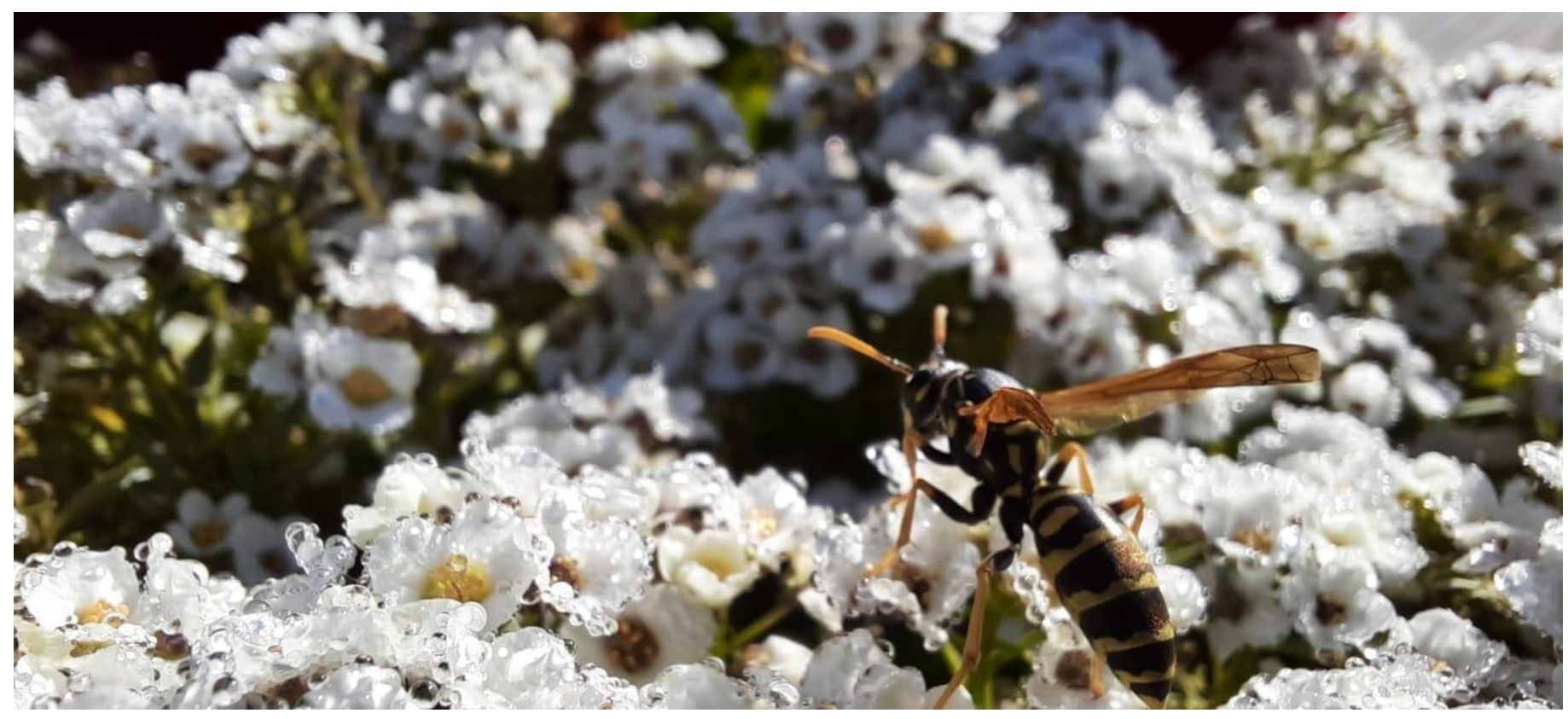

Olesko, Lviv, Ukraine, 2021. Uliana Pidvalna, Lviv, Ukraine 